\title{
An Ultra-Cold Jet of Polarized Atomic Hydrogen
}

\author{
R.S. Raymond, D.G. Crabb, T. Roser \\ University of Michigan, Ann Arbor, MI 48109 \\ G.R. Court \\ Massachussets Institute of Technology, Cambridge, MA 02139 \\ and \\ University of Liverpool
}

\begin{abstract}
Equipment is being tested to determine the applicability of cryogenic atomic physics techniques to the production of dense polarized atomic hydrogen beams. Such beams, with expected densities of $10^{14} / \mathrm{cm}^{3}$, would be useful for internal targets.

Our group is working to determine the applicability of cryogenic atomic physics techniques to the production of polarized atomic hydrogen beams. At low temperatures $(0.5 \mathrm{~K})$ and high magnetic fields $(5 \mathrm{~T})$ the magnetic energy of atomic hydrogen, approximately $\mu_{e} B$, is considerably greater than the thermal energy, $\mathrm{k}$ T. If, then, cold atomic hydrogen approaches a high field region, atoms in the two upper hyperfine states are repelled. Atoms in the lower two states, with electron spins antiparallel to the field, are attracted into the field, where they thermalize by hitting cold walls and are trapped. All cold walls seen by the atoms are coated with ${ }^{4} \mathrm{He}$ to suppress recombination. We shine microwaves onto these trapped atoms in order to drive transitions to the upper hyperfine states. The flipped atoms are then on a potential hill and will be accelerated out of the field forming, we hope, a dense intense beam.

Shown in the figure is our apparatus to test this scheme. The vertical section contains parts of a dilution refrigerator and a liquid nitrogen-temperature dissociator. The horizontal section contains the mixing chamber of the refrigerator and around it, a high-uniformity solenoid. Atoms from the dissociator flow down through the teflon transfer tube and then emerge into the accomodator region, which has ${ }^{4} \mathrm{He}$ coated walls cooled to $0.5 \mathrm{~K}$. The atoms then are guided into the fringe field of the magnet, where electron state selection and trapping
\end{abstract}


occur. Microwaves ( $140 \mathrm{GHz}$ for $5 \mathrm{~T}$ ) enter from the left and atoms expelled to the right form the beam.

Note: Since the CIPAP meeting we have seen signals of atomic hydrogen expelled from the trap when the magnetic field and microwave frequency are matched at either of the allowed atomic transitions. Typically, $10 \mathrm{~mW}$ of microwave power were used. The detector consists of a light copper cup at about $4 \mathrm{~K}$. Atomic hydrogen recombines on the copper with high efficiency and the energy of recombination is measured by the increase in temperature of the detector. Work is continuing to understand loading, trapping, and extraction.

We would like to thank A.D. Krisch, D. Kleppner, and T.J. Greytak for support and advice and T.O. Niinikoski and P.R. Cameron for assitance in the early stages. Brookhaven National Laboratory has provided lab space and cryogenic support. This work is supported by the U.S. Department of Energy.

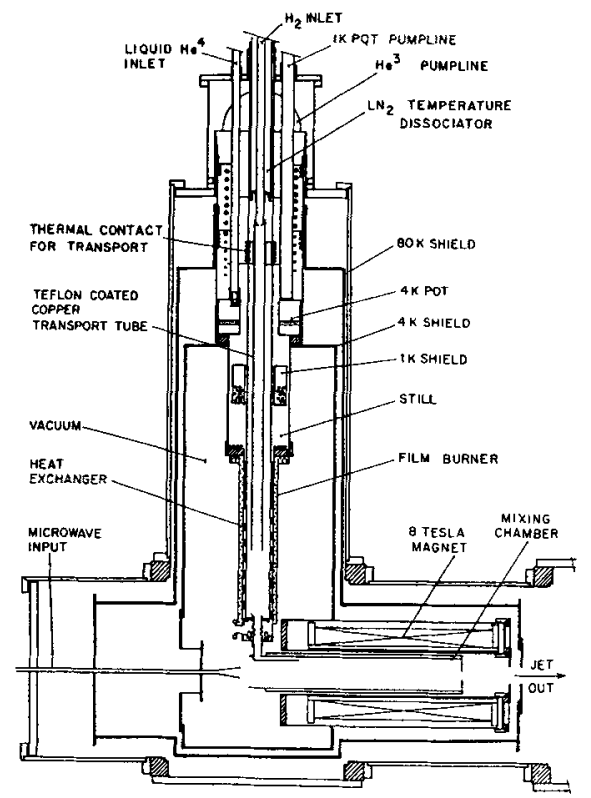

Fig. 1. Polarized jet test apparatus 